\title{
BIBECHANA
}

A Multidisciplinary Journal of Science, Technology and Mathematics

ISSN 2091-0762 (online)

Journal homepage: http://nepjol.info/index.php/BIBECHANA

\section{Bottlenecks to product rate variation problem with batching}

\author{
Shree Ram Khadka \\ Central Department Mathematics,Tribhuvan University, Kathmandu, Nepal \\ P.O.Box, 13143 \\ e-mail: shreeramkhadka@gmail.com \\ Article history: Received 25 May, 2011; Accepted 8 June, 2011
}

\begin{abstract}
The product rate variation problem with batching minimizes the variation in the rate at which different models of a common base product are produced on the assembly lines with the assumption of significant setup and arbitrary processing times for each copy of each model. Establishment of bottlenecks to the problem is important for the feasible and the optimal solution to the problem. In this paper, the lower and the upper bottlenecks to the problem are established. Moreover, small bottlenecks that lead to optimality to some instances are investigated.
\end{abstract}

Keywords: Product rate variation problem; batching; sequencing problem; nonlinear intege programming

\section{Introduction}

The product rate variation problem (PRVP) with batching minimizes the variation in the rate at which different models of a common base product are produced in batches on the assembly lines $[7,8]$. The problem minimizes both the earliness and the tardiness penalties that respond to the customer demands for a variety of models without holding large inventories or incurring large shortages. This is a problem of finding a sequence of batches of different models distributed as evenly as possible on the assembly lines with the assumption of significant set up and arbitrary processing times.

Significant setup and arbitrary processing times can be undertaken when the planning horizon is partitioned into a finite number of time-buckets with equal length. The time length of a time-bucket is called a takt-time. A time-bucket consists of a setup and a batch (a copy or several copies) of a model. The assumption that allows significant

setup and arbitrary processing times forces the product rate variation problem to be a two-phase problem $[7,8]$. We call this problem as the product rate variation problem with batching.

The first phase is the batching problem that determines the batch size and the number of batches of the models. The second phase is the sequencing problem that sequences the batches. The problem that determines the size and the number of batches and minimizes the maximum variation in the rate at which batches of different models of a common base product are produced is called the bottleneck product rate variation with batching problem. 
Shree Ram Khadka / BIBECHANA 8 (2012) 53-58 : BMHSS, p.54

The problem has been formulated as a non-linear integer programming with the objective of minimizing the deviation between the actual and the ideal production under the assumption that the system has sufficient capacity with significant set up and arbitrary processing times each batch of a model is produced in a unit time-bucket $[7,8]$. The problem has mathematically interesting base model with theoretical value and real world applications, see [3].

The problem has been extensively studied and solved in pseudo-polynomial time. The total PRVP i.e. the problem with the objective of minimizing the total deviation has been solved in $O\left(D^{3}\right),[5]$ and the problem with the objective of minimizing the maximum deviation i.e. the bottleneck PRVP in O(DlogD) time [4]. Note that the solution to both cases of the problem is Pareto optimal.

Establishment of bottlenecks is important to the optimization problem since the bottlenecks guaranty whether the optimal or feasible solution exists or not. It is clear that there exists no even feasible solution below the lower bottleneck and every instance has optimal solution below the upper one.

In this paper, the lower and the upper bottlenecks to the problem are established. Moreover, small bottlenecks that lead to optimality to some instances are investigated.

The plan of the paper is as follows. Section 2 reviews the mathematical model. In Section 3, the lower and the upper bottlenecks are established. Section 4 studies small bottlenecks that lead to optimality to some instances are investigated. The last section concludes the paper.

\section{Mathematical Formulation}

Let $s_{i}$ and $p_{i}$ be the setup and processing times of a model $i, i=1, \ldots, n$, respectively. The total demands $\mathrm{D}=\sum_{\mathrm{i}=1}^{\mathrm{n}} \mathrm{d}_{\mathrm{i}}$ are manufactured over the planning horizon $\mathrm{T}$ partitioned into $\mathcal{D}$ time- buckets i.e. the number of batches with the takt-time $t=\frac{T}{D}$. There may exist batches with no models to be manufactured. Such empty batches are potentially useful for the improvement of the system performance [5]. The takt-time satisfies $t \geq s_{i}+p_{i}, i=1, \ldots, n$.

We denote $\tilde{x}_{i k}, i=0,1, \ldots, n ; k=1, \ldots, \mathcal{D}$ to be the actual cumulative number of batches for model $i$ produced during the time-buckets 1 through $\mathrm{k}$. The actual cumulative

production of model $i$ during the same time-buckets is $\gamma_{i} \tilde{x}_{i k}$, where $\gamma_{i}=\frac{d_{i}}{\widetilde{d}_{i}}$ is the average number of copies of model $\mathrm{i}$ per batch. The ideal cumulative production of model $\mathrm{i}$ during 1 through $\mathrm{k}$ timebuckets is $Y_{i} k \tilde{r}_{i}$, where $\tilde{r}_{i}=\frac{\widetilde{d}_{i}}{\mathcal{D}}$ is the batch rate. The sequencing problem minimizes the deviation between the actual and the ideal productions. Let $\mathrm{m}$ be a positive integer.

The mathematical programming for the sequencing phase of the bottleneck product rate variation problem with batching is

subject to

$$
\operatorname{minimize}\left[\mathrm{F}_{\mathrm{m}}=\max _{\mathrm{i}, \mathrm{k}}\left(\mathrm{Y}_{\mathrm{i}}\left|\tilde{\mathrm{x}}_{\mathrm{ik}}-\mathrm{k} \tilde{\mathrm{r}}_{\mathrm{i}}\right|\right)^{\mathrm{m}}\right]
$$

$$
\begin{gathered}
\sum_{\mathrm{i}=1}^{\mathrm{n}} \tilde{\mathrm{x}}_{\mathrm{ik}}=\mathrm{k}, \quad \mathrm{k}=1, \ldots, \mathcal{D} \\
\tilde{\mathrm{x}}_{\mathrm{i}(\mathrm{k}-1)} \leq \tilde{\mathrm{x}}_{\mathrm{ik}}, \quad \mathrm{i}=0,1, \ldots, \mathrm{n} ; \mathrm{k}=2, \ldots, \mathcal{D} \\
\tilde{\mathrm{x}}_{\mathrm{i} \mathcal{D}}=\tilde{\mathrm{d}}_{\mathrm{i}}, \tilde{\mathrm{x}}_{\mathrm{i} 0}=0, \mathrm{i}=0,1, \ldots, \mathrm{n} \\
\tilde{\mathrm{x}}_{\mathrm{ik}} \geq 0, \text { integer } \mathrm{i}=0,1, \ldots, \mathrm{n} ; \mathrm{k}=1, \ldots, \mathcal{D}
\end{gathered}
$$

Constraint (9) ensures that exactly $\mathrm{k}$ batches are produced during the periods 1 through $\mathrm{k}$. Constraint (10) states that the total number of batches is a non decreasing function of k. Constraint (11) guarantees that the batches are exactly met. Constraints (9), (10) and (12) ensure that exactly one batch of a model is sequenced during a unit time-bucket. Note that the formulation of the sequencing phase of the problem is similar to the formulation in [6] for the bottleneck product rate variation problem. 
We denote Problem $\mathrm{F}_{\mathrm{m}}$ for the bottleneck product rate variation problem with batching with the objective function Fm and the constraints (9) to (12).

\section{Bottlenecks}

Establishment of bottlenecks is important to the optimization problem since the bottlenecks guaranty whether the optimal or feasible solution exists or not. It is clear that there exists no even feasible solution below the lower bottleneck and every instance has optimal solution below the upper one.

Let $B$ be a bottleneck to the problem.

Theorem 1. The lower bottleneck to the problem is $\left(\gamma_{i}\left(1-\tilde{r}_{\max }\right)\right)^{m}$.

\section{Proof:}

A batch for some model $\mathrm{i}$ is sequenced at the first time-bucket.

It holds $\min \left(\gamma_{i}\left(1-\tilde{r}_{i}\right)\right)^{m} \leq B$.

For any feasible solution, $\min \left(\gamma_{i}\left(1-\tilde{r}_{i}\right)\right)^{m} \leq B$.

$\Rightarrow\left(\gamma_{i}\left(1-\tilde{r}_{\text {max }}\right)\right)^{m} \leq B$.

Thus, the lower bottleneck is $\left(\gamma_{i}\left(1-\tilde{r}_{\text {max }}\right)\right)^{m}$.

Theorem 2. The upper bottleneck to the problem is $\left(\gamma_{i}\left(1-\frac{1}{\mathcal{D}}\right)\right)^{m}$.

Proof:

For $B=\left(\gamma_{i}\left(1-\frac{1}{\mathcal{D}}\right)\right)^{m},\left\lfloor k_{2} \tilde{r}_{i}+\frac{1}{\gamma_{i}} \sqrt[m]{B}\right\rfloor$

$=\left\lfloor k_{2} \tilde{r}_{i}+1-\frac{1}{D}\right\rfloor$

$=k_{2} \tilde{r}_{i}$, for $k_{2} \tilde{r}_{i}$ being integer.

If $k_{2} \tilde{r}_{i}$ is not an integer,

$\left|k_{2} \tilde{r}_{i}+\frac{1}{\gamma_{i}} \sqrt[m]{B}\right|$

$=\left\lfloor k_{2} \tilde{r}_{i}+1-\frac{1}{\mathcal{D}}\right\rfloor$

$\geq\left\lfloor k_{2} \tilde{r}_{i}\right\rfloor+1$, since $k_{2} \tilde{r}_{i}=\left\lfloor k_{2} \tilde{r}_{i}\right\rfloor+\left(k_{2} \tilde{r}_{i}\right)$, where $\left(k_{2} \tilde{r}_{i}\right)$ is the fractional part of $k_{2} \tilde{r}_{i}$.

$>k_{2} \tilde{r}_{i}$.

Therefore,

$\left|k_{2} \tilde{r}_{i}+\frac{1}{\gamma_{i}} \sqrt[m]{B}\right| \geq k_{2} \tilde{r}_{i}$.

Again, $\left|k_{2} \tilde{r}_{i}-\frac{1}{\gamma_{i}} \sqrt[m]{B}\right|$

$=\left\lceil k_{2} \tilde{r}_{i}-1+\frac{1}{\bar{D}}\right\rceil$.

$=k_{2} \tilde{r}_{i}$, for $k_{2} \tilde{r}_{i}$ being integer.

If $k_{2} \tilde{r}_{i}$ is not an integer,

$\left\lceil k_{2} \tilde{r}_{i}-\frac{1}{\gamma_{i}} \sqrt[m]{B}\right\rceil$

$\left.\leq\left[\mid k_{2} \tilde{r}_{i}\right\rfloor\right]$, since $\left(k_{2} \tilde{r}_{i}\right) \leq 1-\frac{1}{\mathcal{D}}$

$<k_{2} \tilde{r}_{i}$.

Therefore,

$\left.\mid k_{2} \tilde{r}_{i}-\frac{1}{\gamma_{i}} \sqrt[m]{B}\right\rceil \leq k_{2} \tilde{r}_{i}$

Now,

$\sum_{i=1}^{n}\left(\left[k_{2} \tilde{r}_{i}+\frac{1}{\gamma_{i}} \sqrt[m]{\bar{B}}\right]-\left\lceil\left(k_{1}-1\right) \tilde{r}_{i}-\frac{1}{\gamma_{i}} \sqrt[m]{\bar{B}}\right]\right)$

$\geq \sum_{i=1}^{n} k_{2} \tilde{r}_{i}-\sum_{i=1}^{n}\left(k_{1}-1\right) \tilde{r}_{i}$

$\geq k_{2}-k_{1}+1$.

And

$\left.\sum_{i=1}^{n}\left(\mid k_{2} \tilde{r}_{i}-\frac{1}{\gamma_{i}} \sqrt[m]{\bar{B}}\right]-\left\lfloor\left(k_{1}-1\right) \tilde{r}_{i}+\frac{1}{\gamma_{i}} \sqrt[m]{\bar{B}}\right\rfloor\right)$ 
$\leq \sum_{i=1}^{n} k_{2} \tilde{r}_{i}-\sum_{i=1}^{n}\left(k_{1}-1\right) \tilde{r}_{i}$

$\leq k_{2}-k_{1}+1$.

The bottleneck $\left(\gamma_{i}\left(1-\frac{1}{\mathcal{D}}\right)\right)^{m}$ is an upper bottleneck since it satisfies the Hall's theorem [4].

Importance of upper bottleneck is that every instance has an optimal sequence when the given bottleneck is the upper bottleneck. However, it is not guaranteed for smaller value. The upper bottleneck for any instance with $n=1$ is trivially 0 . The lower bottleneck is important because a feasible sequence that exists for lower bottleneck is optimal.

An optimizer always seeks instances that give rise to optimal sequence for smaller bottleneck.

\section{Small Bottleneck}

Every instance has optimal sequence when the given bottleneck is the upper bottleneck. However, it is not guaranteed for smaller value. A feasible solution with a minimum B is optimal. So, it is always interesting and important to seek small bottleneck.

It has been established that there is no instance with a feasible sequence for the bottleneck $B<$ $\left(\frac{Y_{i}}{3}\right)^{m}$.

Theorem 3 No instance with $n \geq 2$ of the problem has a feasible sequence for $B<\left(\frac{\gamma_{i}}{3}\right)^{m}$.

Proof:

The bottleneck $\left(\gamma_{i}\left(1-\tilde{r}_{\text {max }}\right)\right)^{m}$ implies

$1-\tilde{r}_{\text {max }} \leq \frac{1}{\gamma_{i}} \sqrt[m]{B}$.

For feasible sequence, $\widetilde{E}_{m}(i, j) \leq \tilde{L}_{m}(i, j)$, where

$\tilde{E}_{m}(i, j)$ and $\tilde{L}_{m}(i, j)$ are the earliest and the latest sequencing times, respectively.

$\Rightarrow \frac{j-\frac{1}{\gamma_{i}} m_{\sqrt{B}}}{\tilde{r}_{i}} \leq \frac{j-1+\frac{1}{\gamma_{i}} \sqrt[m]{B}}{\tilde{r}_{i}}+1$

$\Rightarrow 1-\tilde{r}_{i} \leq \frac{2}{\gamma_{i}} \sqrt[m]{B}$ for all $i$.

$\Rightarrow 1-\tilde{r}_{\min } \leq \frac{2}{\gamma_{i}} \sqrt[m]{B}$

$\Rightarrow \sum_{i=1}^{n} \tilde{r}_{i} \leq \frac{2}{\gamma_{i}} \sqrt[m]{B}, \tilde{r}_{i} \neq \tilde{r}_{\text {min }}$

$\Rightarrow \tilde{r}_{\text {max }} \leq \sum_{i=1}^{n} \tilde{r}_{i} \leq \frac{2}{\gamma_{i}} \sqrt[m]{B}$

$\Rightarrow 1-\tilde{r}_{\text {max }} \geq 1-\frac{2}{\gamma_{i}} \sqrt[m]{B}$.

Then, $1-\frac{2}{\gamma_{i}} \sqrt[m]{B} \leq 1-\frac{1}{\gamma_{i}} \sqrt[m]{B}$

$\Rightarrow \frac{\gamma_{i}}{3} \leq \sqrt[m]{B}$.

This shows that any instance with the bottleneck less than $\left(\frac{\gamma_{i}}{3}\right)^{m}$ does not yield any feasible sequence.

For any feasible solution, $\left(\gamma_{i\left|\left[k \tilde{r}_{i}\right]-k \tilde{r}_{i}\right|}\right)^{m} \leq\left(\gamma_{i\left|\tilde{x}_{i k}-k \tilde{r}_{i}\right|}\right)^{m}, i=1, \ldots, n$, where $\left[k \tilde{r}_{i}\right]$ is the closest integer to $k \tilde{r}_{i}$. It is observed that $\left|\left[k \tilde{r}_{i}\right]-k \tilde{r}_{i}\right|=\frac{1}{2}$ if $\mathcal{D}$ is even and $\left|\left[k \tilde{r}_{i}\right]-k \tilde{r}_{i}\right|=\frac{\mathcal{D}-1}{2 \mathcal{D}}$ if $\mathcal{D}$ is odd [1]. This shows that any instance, $n \geq 2$, with even $\mathcal{D}$ of the problem has lower bottleneck $\left(\frac{\gamma_{i}}{2}\right)^{m}$ and has lower bottleneck $\left(\frac{\gamma_{i}}{3}\right)^{m}$ for the instance, $n \geq 2$, with odd $\mathcal{D}$.

For $n \geq 2$, a standard instance $\left(1,2,2^{2}, \ldots, 2^{i-1}\right), i=1, \ldots, n$, has optimal sequence with bottleneck less than $\left(\frac{\gamma_{i}}{2}\right)^{m}[2]$.

Theorem 4 The instance $\left(1,2,2^{2}, \ldots, 2^{i-1}\right), i=1, \ldots, n, n \geq 2$ has an optimal sequence with $B<\left(\frac{\gamma_{i}}{2}\right)^{m}$. 


\section{Proof:}

We consider the bottleneck $B=\left(\gamma_{i}\left(\frac{1}{2}-\frac{1}{2 D}\right)\right)^{m}$ and assume that $(i, j)$ be sequenced in the ideal position $\left\lceil\frac{2 j-1}{2 \tilde{r}_{i}}\right\rceil$.

For this instance, $B=\left(\gamma_{i}\left(\frac{2^{n-1}-1}{2^{n}-1}\right)\right)^{m}<\left(\frac{\gamma_{i}}{2}\right)^{m}$ and

$\left\lceil\frac{2 j-1}{2 \tilde{r}_{i}}\right\rceil=2^{n-i}(2 j-1), i=1, \ldots, n ; j=1, \ldots, \tilde{d}_{i}$.

We show that the instance has a feasible sequence with this bottleneck.

Consider, $\frac{j-\frac{1}{\gamma_{i}} \sqrt[m]{\bar{B}}}{\tilde{r}_{i}}=\frac{j-\frac{2^{n-1}-1}{2^{n}-1}}{\frac{2^{2}-1}{2^{n}-1}}$

$=2^{n-i}(2 j-1)+\frac{1-(2 j-1)}{2^{i}}$

$\leq 2^{n-i}(2 j-1)$

$=\frac{j-1+\frac{2^{n-1}-1}{2^{n-1}}}{\frac{2^{i-1}}{2^{n-1}}}+1$

$=\frac{j-1+\frac{1}{\gamma_{i}} \sqrt[m]{B}}{\widetilde{r}_{i}}+1$

One can write $\widetilde{E}_{m}(i, j)$

$\leq 2^{n-i}(2 j-1)$

$\leq \tilde{L}_{m}(i, j)$, since $2^{n-i}(2 j-1)$ is an integer.

Thus, the instance has a feasible sequence to the problem.

Now, we show that $(i, j)$ does not compete with $(i, j)$ with $i \neq i$ and $j \neq j$.

Assume that $(i, j)$ and $(i, j)$ be sequenced at the same position.

$\Rightarrow\left\lceil\frac{2 j-1}{2 \tilde{r}_{i}}\right\rceil=\left\lceil\frac{2 j-1}{2 \tilde{r}_{i}}\right\rceil$

$\Rightarrow\left\lceil\frac{(2 j-1)\left(2^{n}-1\right)}{2^{i}}\right\rceil=\left\lceil\frac{(2 j-1)\left(2^{n}-1\right)}{2^{i}}\right\rceil$, for $\tilde{d}_{i}=2^{i-1}, i=1, \ldots, n$.

$\Rightarrow\left\lceil(2 j-1) 2^{n-i}-(2 j-1) 2^{-i}\right\rceil=\left\lceil(2 j-1) 2^{n-i}-(2 j-1) 2^{-i}\right\rceil$.

$\Rightarrow \frac{2 j-1}{2^{i}}=\frac{2 j-1}{2^{i}}$

Since both $(2 j-1)$ and $(2 j-1)$ are odd, neither $2^{i}$ divides $(2 j-1)$ nor $2^{i}$ divides $(2 j-1)$.

This implies $i=i$ and $=j$.

Thus, the copies do not compete one another for the position. A feasible sequence of which the copies do not compete one another for the position is optimal.

\section{Conclusion}

Establishment of bottlenecks is important to the optimization problem since the bottlenecks guaranty whether the optimal or feasible solution exists or not. It is clear that there exists no even feasible solution below the lower bottleneck and every instance has optimal solution below the upper one.

The lower and the upper bottlenecks to the problem has been established to be $\left(\gamma_{i}\left(1-\tilde{r}_{\text {max }}\right)\right)^{m}$ and $\left(\gamma_{i}\left(1-\frac{1}{\mathcal{D}}\right)\right)^{m}$, respectively. It has been investigated that the only instance with optimality for the bottleneck less than $\left(\frac{Y_{i}}{2}\right)^{m}$ is $\left(1,2,2^{2}, \ldots, 2^{i-1}\right), i=1, \ldots, n, n \geq 2$. Further, there exists no such instance for the bottleneck less than $\left(\frac{\gamma_{i}}{3}\right)^{m}$.

\section{References}

[1] N. Brauner, and Y. Crama, Discrete Applied Mathematics, 134 (2004), 25.

[2] N. Brauner, V. Jost and W. Kubiak, On symmetric Fraenkel's and small deviations conjecture, Les Cahiers du Laboratoire Leibniz-IMAG, 54, Grenoble, France, 2004.

[3] T. N. Dhamala and S. R. Khadka, Iranian Journal of Optimization, 1, 3, (2009)266.

[4] S. R. Khadka and T. N. Dhamala, Iranian Journal of Optimization, 3 (2010) 477.

[5] W. Kubiak and M. Yavuz, Manufacturing and Service Operations Management, 10, 3, (2008) 506. 
Shree Ram Khadka / BIBECHANA 8 (2012) 53-58 : BMHSS, p.58

[6] G. Steiner and J. S. Yeomans, Management Science, 39, 6, (1993)728.

[7] M. Yavuz, E. Akcali and S. Tufekci, International Journal of Production Research, 44, 15, (2006)3061.

[8] M. Yavuz and S. Tufekci, International Journal of Production Research, 45, 17, (2007) 389 